\title{
Macrocystic Neoplasms of the Pancreas: CT Differentiation of Serous Oligocystic Adenoma from Mucinous Cystadenoma and Intraductal Papillary Mucinous Tumor
}

\author{
Sang Youn Kim ${ }^{1}$ \\ Jeong Min Lee ${ }^{1}$ \\ Se Hyung Kim ${ }^{1}$ \\ Kyung-Sook Shin ${ }^{1,2}$ \\ Young Jun Kim ${ }^{1}$ \\ Su Kyung $A n^{1}$ \\ Chang Jin Han ${ }^{1}$ \\ Joon Koo Han ${ }^{1}$ \\ Byung Ihn Choi ${ }^{1}$
}

Keywords: abdominal imaging, CT, intraductal papillary mucinous tumor, macrocystic serous adenoma, mucinous cystadenoma, pancreas, pancreaticobiliary imaging, serous oligocystic adenoma

\section{DOI:10.2214/AJR.05.0337}

Received February 27, 2005; accepted after revision June 17, 2005.

\section{'Department of Radiology and Institute of Radiation} Medicine, Seoul National University Hospital and College of Medicine, 28 Yongon-dong, Chongno-gu, Seoul 110-744, Korea. Address correspondence to J. K. Han.

2Present address: Department of Radiology, Chungnam National University Hospital, Seoul, Korea.

\section{CME}

This article is available for CME credit. See www.arrs.org for more information.

\section{AJR 2006; 187:1192-1198}

0361-803X/06/1875-1192

(C) American Roentgen Ray Society
OBJECTIVE. The purpose of our study was to determine useful CT criteria for differentiating serous oligocystic adenomas of the pancreas from other similarly presenting neoplasms, such as mucinous cystadenoma and intraductal papillary mucinous tumor of the branch duct type.

MATERIALS AND METHODS. Forty-one patients with histologically confirmed macrocystic neoplasms of the pancreas were enrolled: serous oligocystic adenoma in 10 patients, mucinous cystadenoma in 13 , and intraductal papillary mucinous tumor in 18 . Location, greatest dimension, shape, presence of mural nodules, presence of wall calcification, and the extent and degree of main pancreatic duct (MPD) dilatation were analyzed with CT. The lesions were categorized into seven groups according to their shapes: multicystic, lobulated contour with and without internal septation, smooth contour with and without internal septation, pleomorphic cystic, and clubbed fingerlike cystic. Comparative studies were performed using Fisher's exact test and the Mann-Whitney $U$ test.

RESULTS. Significant differences in lesion shape were found between serous oligocystic adenoma and the other macrocystic neoplasms (mucinous cystadenoma $[p<0.05]$, intraductal papillary mucinous tumor $[p<0.05])$. Serous oligocystic adenoma had a multicystic or lobulated contour with or without septation, whereas mucinous cystadenoma had a smooth contour with or without septation and intraductal papillary mucinous tumor had either a pleomorphic or a clubbed fingerlike cystic shape. Serous oligocystic adenoma showed proximal MPD dilatation from the lesion, whereas intraductal papillary mucinous tumor showed distal or whole MPD dilatation $(p<0.05)$. No significant difference was apparent among the three diseases in terms of location, greatest dimension, or presence of calcification or mural nodules.

CONCLUSION. Serous oligocystic adenoma of the pancreas has characteristic CT findings that differentiate it from other cystic tumors. It appears as a multicystic or lobulated cystic lesion with septation.

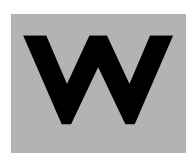
ith the widespread use of abdominal CT, cystic neoplasms of the pancreas are being increasingly recognized, but their accurate characterization and differentiation remain difficult because of their overlapping morphologies. Among them, the serous pancreatic cystadenoma is a common benign neoplasm. The most common variety of serous cystadenoma is the serous microcystic adenoma. The other, much less common variant of serous cystadenoma is serous oligocystic adenoma, which has fewer but much larger cysts that are histologically similar to those of serous microcystic adenoma $[1,2]$. Many authors have reported that the incidence of serous oligocystic adenoma ranges from $10 \%$ to $25 \%$ of serous pancreatic cystadenomas [3-5].
Pathologists say that the macroscopic approach to diagnosis makes it easy to differentiate serous oligocystic adenoma from serous microcystic adenoma because the latter macroscopically tends to be a spongelike cystic dal epithelial cells. The question is whether serous oligocystic adenomas have macroscopic findings similar to other macrocystic tumors such as mucinous cystadenoma or intraductal papillary mucinous tumor. Since the World Health Organization subclassified serous macrocystic adenoma (a synonym for serous oligocystic adenoma) as a subgroup of pancreatic serous cystic tumors, for example, many authors have reported that several cases of serous oligocystic adenoma have been misdiagnosed as mucinous cystadenoma and inappropriately managed [6-16]. lesion lined by uniform glycogen-rich cuboi- 


\section{CT of Pancreatic Neoplasms}

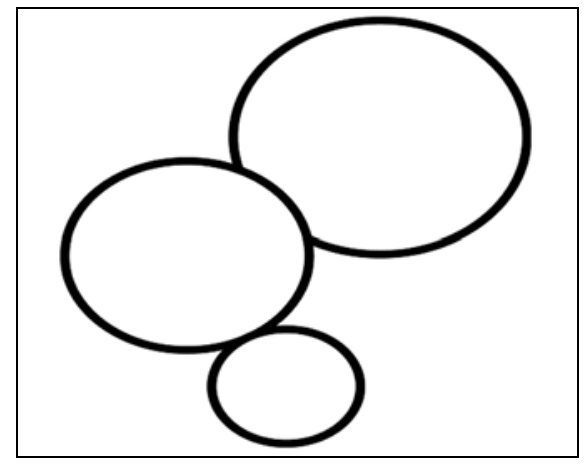

A

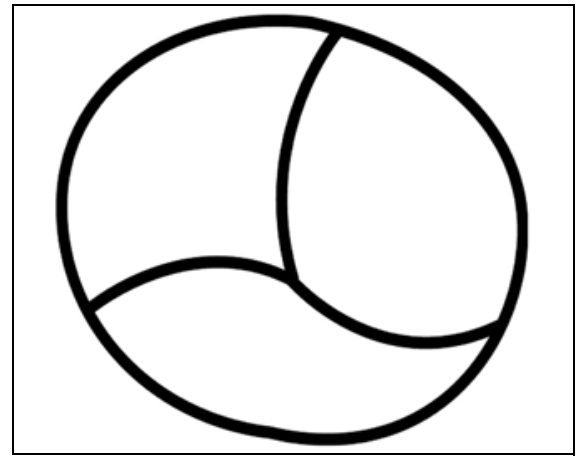

D

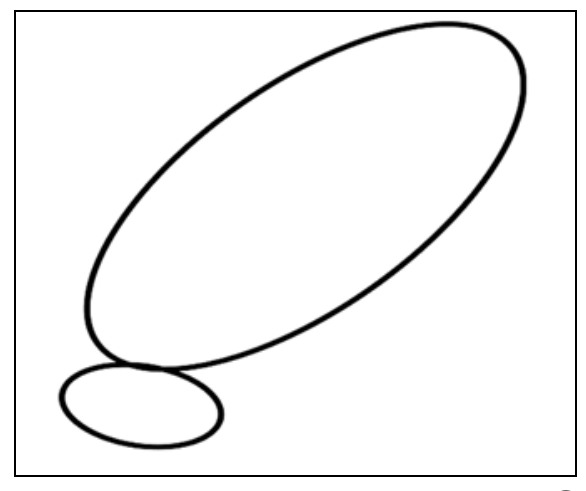

G

Because each pancreatic cystic neoplasm has different malignant potential, it is clinically valuable to determine characteristic imaging findings that can enable the differentiation of serous oligocystic adenoma from other macrocystic neoplasms. Mucinous cystadenomas span the spectrum of malignant potential, with serous cystadenomas representing the most benign cystadenomas. Intraductal papillary mucinous tumors of the pancreas mimic mucinous cystadenomas and have similar biologic behavior $[11,12,17,18]$. Thus, the purpose of this study was to describe the typical CT findings of serous oligocystic adenoma of the pancreas and to identify features
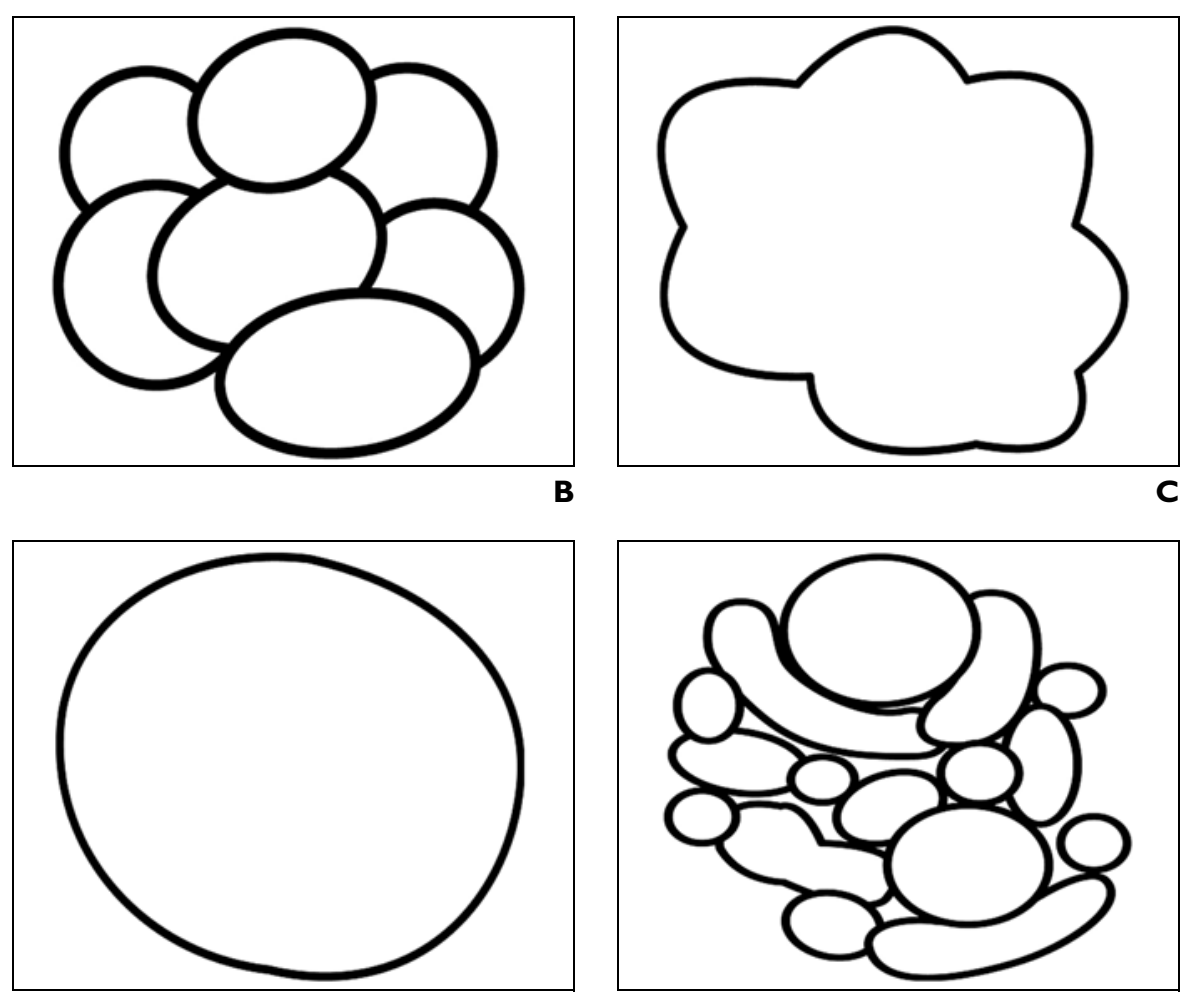

Fig. 1-Schematic diagrams of shapes of cystic lesions

A, "Multicystic shape" was defined as conglomeration of two or more round cysts. We defined a cyst as a simple closed curve without concavity to differentiate it from an internal locule in septate shape.

B, "Lobulated shape with septation" was defined as a septate simple closed curve that cannot be described as borders of the same circle. "Internal locule" was defined as an angular closed curve without protrusion from border of a primary lesion.

C, "Lobulated shape without septation" was defined as a nonseptate simple closed curve that cannot be described as borders of the same circle.

D, "Smooth shape with septation" was defined as a septate simple closed curve with borders of the same circle and internal septation.

E, "Smooth shape without septation" was defined as a nonseptate simple closed curve with borders of the same circle.

F, "Pleomorphic cystic shape" was defined as composed of three or more cysts that include more than one oval or tubular cyst. We defined a "tubular" or "oval" cyst as one having a conic section, the plane of which was not parallel to axis, base, or generatrix of the intersected cone.

G, “Clubbed fingerlike cystic shape" was defined as one containing one or two cysts with a tubular or oval shape.

differentiating this disease from other similarly presenting macrocystic neoplasms such as mucinous cystadenoma and intraductal papillary mucinous tumor.

\section{Materials and Methods \\ Data Collection}

We searched the pathology database of our hospital for cases of serous oligocystic adenoma, mucinous cystadenoma, and intraductal papillary mucinous tumor of the pancreas during the period January 1996 to June 2003. Terms searched for were "serous oligocystic adenoma and pancreas," "macrocystic serous adenoma and pancreas," "serous cystadenoma and pancreas," "mucinous cys- tadenoma and pancreas," and "branch duct type intraductal papillary mucinous tumor and pancreas." All lesions were confirmed at histopathology of samples obtained by pancreatic surgery or biopsy.

Sixty patients were identified as having one of these three pancreatic macrocystic neoplasms. Seventeen were excluded because of the nonavailability of appropriate CT images. Thus, 43 patients were selected for pathologic review. An experienced pathologist reconfirmed the diagnoses by microscopic review of pathology slides. However, as a result of the pathologic review, two patients were excluded because their tumors were microcystic serous cystadenomas, even if they were included initially because their initial patho- 


\section{Kim et al.}

Fig. 2-Serous oligocystic adenoma of pancreas in 49-year-old man. Contrast-enhanced CT scan obtained during portal venous phase shows multicystic shaped cystic mass (arrow) in neck of pancreas. Proximal main pancreatic duct dilatation was also noted.

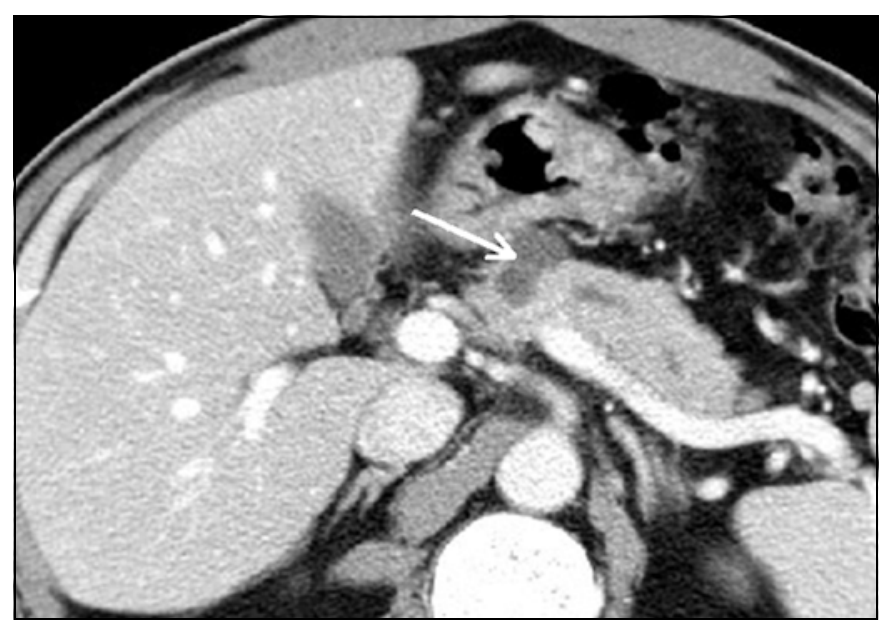

logic diagnosis was serous cystadenoma. Therefore, 41 patients were finally enrolled. These comprised 13 men and 28 women (mean age, 54 years; age range, 32-77 years). The final diagnoses were serous oligocystic adenoma in 10 patients (age range, 34-70 years; mean age, 48 years; 2 men, 8 women), mucinous cystadenoma in 13 (age range, 32-77 years; mean age, 49 years; all women), and intraductal papillary mucinous tumor in 18 (age range, 49-74 years; mean age, 60 years; 11 men, 7 women).

\section{CT Acquisition}

CT examinations of the 41 patients were performed using a Somatom Plus-4 scanner (Siemens Medical Solutions), a HighSpeed Advantage scanner (GE Healthcare), or an MX8000 4-MDCT scanner (Philips Medical Systems) at our institution. After unenhanced CT was performed, each patient received $120 \mathrm{~mL}$ of a nonionic contrast material ([iopromide] Ultravist 370 , Schering) through an 18-gauge angiographic catheter inserted into a forearm vein. CT images were routinely obtained during full inspiration with the patient in a supine position. Contrast material was injected at $3 \mathrm{~mL} / \mathrm{s}$ using an automatic power injector.

Helical CT was performed using a single-detector scanner and the following parameters: 5-mm collimation, 1:1 table pitch, and 5-mm reconstruction intervals. If an MDCT scanner was used, the parameters were $2.5-\mathrm{mm}$ detector collimation, 20 $\mathrm{mm} / \mathrm{s}$ table speed, 3.2- $\mathrm{mm}$ slice thickness, and 1.6$\mathrm{mm}$ reconstruction interval. Unenhanced $\mathrm{CT}$ was performed using the same parameters. Multiphasic helical CT scans were obtained at 30 seconds (arterial phase), at 40 seconds (pancreatic phase), and at 70 seconds (portal venous phase) after the initiation of the contrast injection.

\section{CT Imaging Analysis}

Two experienced abdominal radiologists who were blinded to the specific diagnoses and clinical information reviewed the CT images by consensus in terms of the following morphologic features of the lesions: location (head, neck, body, or tail), greatest dimension (measured in centimeters), presence of mural nodules (present or absent), presence of wall calcification (present or absent), extent of main pancreatic duct (MPD) dilatation (none, diffuse, distal to, or proximal from the tumor), degree of MPD dilatation (normal, mild, moderate, or severe), and tumor shape. All CT scans were reviewed on a PACS workstation.

The presence of wall calcification was determined using unenhanced images. The greatest diameter of the tumor and the extent of MPD dilatation were measured with images obtained during the portal venous phase by one of the reviewers. The degree of MPD dilatation was considered mild if the diameter was more than $2 \mathrm{~mm}$, moderate if more than $4 \mathrm{~mm}$, and severe if more than $6 \mathrm{~mm}$. CT images of axially reconstructed sectional planes were reviewed.

\section{Categorization of Shape}

To identify morphologic features that can be used to differentiate these tumors, tumor shape was categorized as multicystic, lobulated contour with or without internal septation, smooth contour with or without internal septation, pleomorphic cystic, and clubbed fingerlike cystic. A "multicystic shape" was defined as a conglomeration of two or more round evenly sized cysts (Fig. 1A). A "lobulated shape" was defined as the shape of a simple closed curve that could not be described as the borders of the same circle (Figs. 1B and 1C). A "smooth shape" was a simple closed curve with the borders of the same circle (Figs. 1D and 1E).
In order to differentiate the multicystic and multilobulated shapes with internal septation, a "pleomorphic cystic shape" was defined as one containing three or more cysts that should include more than one oval or tubular cyst [1] (Fig. 1F). A tubular or oval-shaped cyst was defined as having a conic section with a plane that was not parallel to the axis, base, or generatrix of the intersected cone differentiating it from a round cyst. Finally, a "clubbed fingerlike cystic shape" was defined as one having one or two tubular or oval cysts (Fig. 1G).

Because we needed to differentiate the lobulated type with septation from the multicystic, pleomorphic cystic, and clubbed fingerlike cystic shapes, we defined an internal locule, seen in the lobulated and smooth shapes with septation, as an angular closed curve without protrusion from the border of a main lesion, whereas we defined a cyst as a simple closed curve without concavity.

\section{Statistical Analysis}

The mean longest diameters of each type of lesion were compared using the Mann-Whitney $U$ test. Fisher's exact test was used to compare other morphologic features. A $p$ value of less than 0.05 was considered to indicate a statistically significant difference. Because we wanted to identify features capable of differentiating serous oligocystic adenoma of the pancreas from other cystic tumors of the pancreas, statistical analysis was not performed for comparisons between mucinous cystadenoma and intraductal papillary mucinous tumor.

\section{Results}

Serous oligocystic adenomas ranged in longest diameter from 2.0 to $7.5 \mathrm{~cm}$ (average, $4.0 \pm 1.65 \mathrm{~cm})$. In $8(80 \%)$ of 10 patients, lesions were located in the body or the tail of the pancreas. No lesion had mural nodules or wall calcification. In terms of the extent and the degree of MPD dilatation, 8 serous oligocystic adenomas $(80 \%)$ were not accompanied by MPD dilatation. Of the 7 shape categories, 6 serous oligocystic adenomas $(60 \%)$ had a lobulated shape (septate, 4 ; nonseptate, 2) and 3 serous oligocystic adenomas (30\%) had a multicystic shape (Figs. 2 and 3; Tables 1 and 2).

Mucinous cystadenomas ranged from 2.0 to $12.5 \mathrm{~cm}$ (average, $6.07 \pm 3.27 \mathrm{~cm}$ ). Eleven $(84.6 \%)$ of the 13 cysts were located in the tail of the pancreas. Twelve patients $(92.3 \%)$ had no mural nodules, and 4 (30.8\%) had wall calcification. Three patients $(23.1 \%)$ showed MPD dilatation (2, mild proximal dilatation; 1 , diffuse moderate dilatation). In terms of shape, 12 cysts $(92.3 \%)$ had a smooth shape (septate, 7; nonseptate, 5) (Fig. 4; Tables 1 and 2). 


\section{CT of Pancreatic Neoplasms}
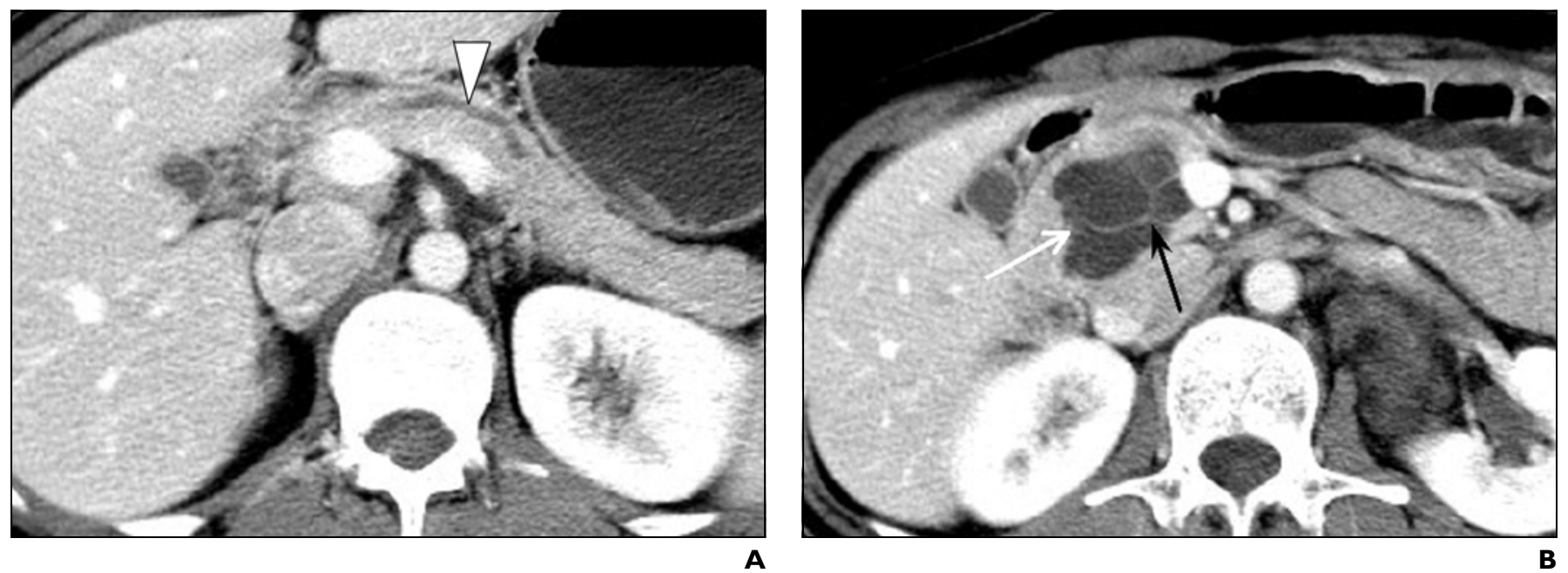

Fig. 3-Serous oligocystic adenoma of pancreas in 34-year-old woman.

A and B, Contrast-enhanced CT scans obtained during portal venous phase show lobulated cystic mass in head of pancreas (white arrow, B). Mass has many internal locules and subtle enhancing septa (black arrow, B). Proximal main pancreatic duct dilatation (arrowhead, A) is also noted.

When serous oligocystic adenoma was compared with mucinous cystadenoma, serous oligocystic adenoma showed a statistically significant difference in lesion shape $(p=0.001)$. Serous oligocystic adenoma tended to have the multicystic or the lobulated shape (Figs. 2 and 3). On the other hand, mucinous cystadenoma tended to have a smooth shape (Table 1). Tumor location $(p=0.108)$, greatest dimension $(p=0.111)$, presence of mural nodules $(p=1.0)$, presence of wall calcification $(p=0.104)$, and extent $(p=$ $1.0)$ and degree $(p=0.476)$ of MPD dilatation were statistically inadequate for differentiating serous oligocystic adenoma from mucinous cystadenoma (Table 2).

TABLE I: Shapes of Three Types of Macrocystic Pancreatic Tumors in 4 I Patients

\begin{tabular}{l|c|c|c}
\hline \multirow{2}{*}{\begin{tabular}{l} 
Shape \\
\cline { 2 - 4 }
\end{tabular}} & $\begin{array}{c}\text { Serous Oligocystic } \\
\text { Adenoma } \\
(n=10)\end{array}$ & $\begin{array}{c}\text { Mucinous } \\
\text { Cystadenoma } \\
(n=13)\end{array}$ & $\begin{array}{c}\text { Intraductal Papillary } \\
\text { Mucinous Tumor } \\
(n=18)\end{array}$ \\
\hline $\begin{array}{l}\text { Multicystic } \\
\text { Lobulated }\end{array}$ & $3(30)$ & 0 & $2(11.1)$ \\
$\quad$ Septate & $4(40)$ & $1(7.8)$ & $1(5.6)$ \\
$\quad$ Nonseptate & $2(20)$ & 0 & 0 \\
Smooth & 0 & $7(53.8)$ & 0 \\
Septate & $1(10)$ & $5(38.5)$ & $1(5.6)$ \\
$\quad$ Nonseptate & 0 & 0 & $10(55.6)$ \\
Pleomorphic cystic & 0 & 0 & $4(22.2)$ \\
Clubbed fingerlike cystic & $10(100)$ & $13(100)$ & $18(100)$ \\
\hline Total & & & \\
\hline
\end{tabular}

Note-Numbers in parentheses are percentages.
Intraductal papillary mucinous tumors ranged from 1.0 to $5.1 \mathrm{~cm}$ (average, $3.09 \pm$ $1.25 \mathrm{~cm})$. In $12(66.7 \%)$ of 18 patients, cystic lesions were located in the body or the tail of the pancreas, and in 5 patients $(27.8 \%)$ lesions were located in the head of the pancreas. Only one intraductal papillary mucinous tumor $(5.6 \%)$ had mural nodules, and only $3(16.7 \%)$ had wall calcification. Six patients $(33.3 \%)$ had diffuse MPD dilatation. In terms of shape, 10 lesions $(55.6 \%)$ showed the pleomorphic cystic shape. Four lesions (22.2\%) had the clubbed fingerlike cystic shape (Figs. 5 and 6; Tables 1 and 2).
When we compared serous oligocystic adenomas with intraductal papillary mucinous tumors, statistically significant differences were observed in the shape and the extent of MPD dilatation (Table 3). Serous oligocystic adenomas tended to present the multicystic or the lobulated shape. On the other hand, intraductal papillary mucinous tumors tended to have the pleomorphic cystic or the clubbed fingerlike cystic shape $(p=0.001)$ (Table 1). The majority of serous oligocystic adenomas and intraductal papillary mucinous tumors showed no MPD dilatations. However, when dilatation was observed, intraductal papillary mucinous tumors showed diffuse or distal MPD dilatation (diffuse, 6/18; distal, 2/18; none, 10/18), whereas serous oligocystic adenomas showed proximal MPD dilatation $(2 / 10)(p=0.025)$. Tumor location $(p=0.751)$, greatest dimension $(p=0.194)$, presence of mural nodules $(p=1.0)$, presence of wall calcification ( $p=0.533)$, and degree of MPD dilatation $(p=0.525)$ were statistically inadequate for differentiating serous oligocystic adenoma from intraductal papillary mucinous tumor (Table 2).

\section{Discussion}

The potential malignant degeneration of the serous oligocystic adenoma is low. Therefore, some authors advise that patients who are not clinically suited for surgical treatment can be closely followed [1, 2, 19]. However, serous oligocystic adenomas can be easily misdiagnosed as another macrocystic neoplasm, such as mucinous cystadenoma or intraductal papil- 


\section{Kim et al.}

lary mucinous tumor on preoperative imaging studies, and treated with surgical resection. Indeed, specific CT features of serous oligocystic

adenoma that helpfully differentiate it from other cystic tumors of the pancreas are not well described in the literature.

\section{TABLE 2: Other CT Features of the Three Types of Macrocystic Pancreatic} Tumors

\begin{tabular}{|c|c|c|c|}
\hline \multirow[b]{2}{*}{ CT Feature } & \multicolumn{3}{|c|}{ Type } \\
\hline & $\begin{array}{c}\text { Serous Oligocystic } \\
\text { Adenoma } \\
(n=10)\end{array}$ & $\begin{array}{c}\text { Mucinous } \\
\text { Cystadenoma } \\
(n=13)\end{array}$ & $\begin{array}{c}\text { Intraductal Papillary } \\
\text { Mucinous Tumor } \\
(n=18)\end{array}$ \\
\hline Mean of longest dimension $(\mathrm{cm})^{\mathrm{a}}$ & $4.0 \pm 1.65$ & $6.07 \pm 3.27$ & $3.09 \pm 1.25$ \\
\hline Location & & & \\
\hline Head & $1(10)$ & $2(15.4)$ & $5(27.8)$ \\
\hline Neck & $1(10)$ & 0 & $1(5.6)$ \\
\hline Body & $3(30)$ & 0 & $5(27.8)$ \\
\hline Tail & $5(50)$ & $11(84.6)$ & $7(38.9)$ \\
\hline \multicolumn{4}{|l|}{ Mural nodule } \\
\hline Present & 0 & $1(7.7)$ & $1(5.6)$ \\
\hline Absent & $10(100)$ & 12 (92.3) & $17(94.4)$ \\
\hline \multicolumn{4}{|l|}{ Wall calcification } \\
\hline Present & 0 & $4(30.8)$ & $3(16.7)$ \\
\hline Absent & $10(100)$ & $9(69.2)$ & $15(83.3)$ \\
\hline \multicolumn{4}{|l|}{ Extent of MPD dilatation } \\
\hline Proximal & $2(20)$ & $2(15.4)$ & 0 \\
\hline Distal & 0 & 0 & $2(11.1)$ \\
\hline Whole & 0 & $1(7.7)$ & $6(33.3)$ \\
\hline None & $8(80)$ & $10(76.9)$ & $10(55.6)$ \\
\hline \multicolumn{4}{|l|}{ Degree of MPD dilatation ${ }^{b}$} \\
\hline Normal & $8(80)$ & $10(76.9)$ & $10(55.6)$ \\
\hline Mild & 0 & $2(15.4)$ & $3(16.7)$ \\
\hline Moderate & $2(20)$ & $1(7.7)$ & $5(27.8)$ \\
\hline Severe & 0 & 0 & 0 \\
\hline
\end{tabular}

Note-MPD = main pancreatic duct. Numbers in parentheses are percentages.

${ }^{\text {a }}$ Measured on CT.

${ }^{\mathrm{b}}<2 \mathrm{~mm}=$ normal, $2-4 \mathrm{~mm}=$ mild, $4-6 \mathrm{~mm}=$ moderate,$>6 \mathrm{~mm}=$ severe.

Fig. 4-Mucinous cystadenoma of pancreas in 46-year-old woman. Contrastenhanced CT scan obtained during pancreatic phase shows smooth cyst mass in tail of pancreas (white arrow). Mass has many internal locules with septa (black arrow).

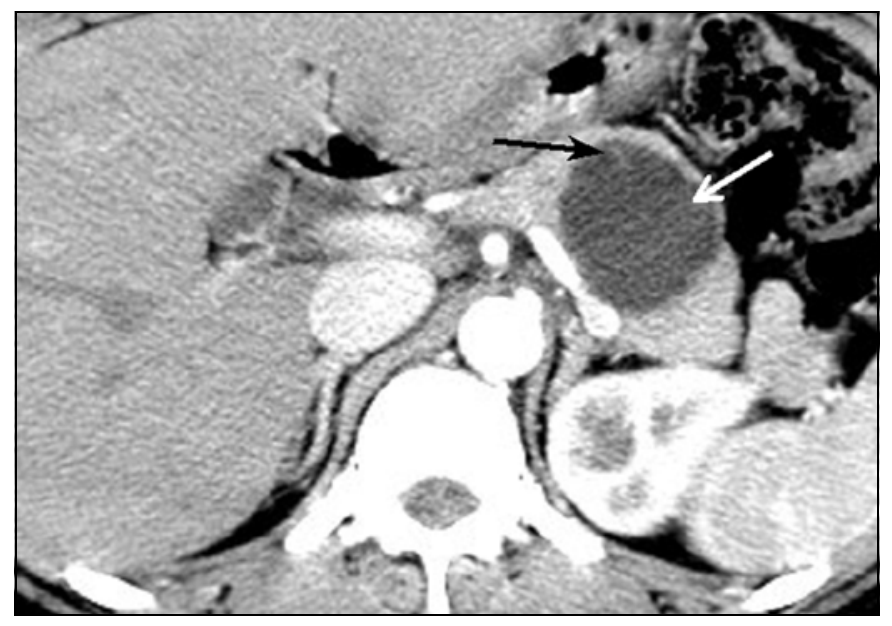

Several authors have attempted to identify radiologic findings capable of differentiating serous oligocystic adenoma from other macrocystic neoplasms of the pancreas and have reported the limited value of $\mathrm{CT}$ for this purpose $[1,2,6-8,10-13,15,16]$. However, those previous reports were limited because detailed CT findings such as tumor shape or accompanying MPD dilatation were not considered during image analysis. More recently, Cohen-Scali et al. [14] found that CT was helpful for differentiating serous oligocystic adenoma and mucinous cystadenoma, and they described specific CT findings indicating serous oligocystic adenoma-namely, location in the pancreatic head, a lobulated contour, and the absence of wall enhancement. However, those authors did not include intraductal papillary mucinous tumors in their study population. On the basis of our clinical experiences, serous oligocystic adenoma is sometimes misdiagnosed as intraductal papillary mucinous tumor, especially as the branch duct type. Thus, we included intraductal papillary mucinous tumor as a subgroup.

In this study, we found that lesion shape was the most useful feature among the seven imaging features for the differential diagnosis of the three types of cystic neoplasms of the pancreas. The multicystic and lobulated shapes were prevalent in serous oligocystic adenomas, the smooth shape in mucinous cystadenomas, and the pleomorphic cystic and the clubbed fingerlike cystic shapes in branch duct type intraductal papillary mucinous tumors. This result is in accord with the findings of Santos et al. [20] regarding serous oligocystic adenoma, in which serous oligocystic adenomas were most commonly found to be composed of a few cysts and to contain tiny cysts that were irregularly arranged and separated by mostly broad septa. The key morphologic feature of intraductal papillary mucinous tumor can be considered to be a presentation of branch pancreatic duct dilatation. In our study, dilated branch pancreatic ducts appeared as clusters of small cysts with a grapelike appearance, or as a multilocular cyst with papillary projections, or as a single cystic lesion with lobulated or irregular margins communicating with a dilated or normal MPD. This result is in accordance with the findings of previous studies $[17,18]$.

Of the seven imaging features, the extent of MPD dilatation was also an important morphologic feature that allowed differential diagnosis between serous oligocystic adenoma and branch duct type intraductal pap- 


\section{CT of Pancreatic Neoplasms}

illary mucinous tumor. If combined with dilated MPD, diffuse or distal MPD dilatation was exclusively observed in intraductal papillary mucinous tumor, whereas proxi- mal MPD dilatation tended to be observed in serous oligocystic adenoma. Diffuse MPD dilatation in intraductal papillary mucinous tumor was mostly associated with mucin se- creted from the tumor, whereas proximal MPD dilatation in serous oligocystic adenoma is probably a mass effect due to extrinsic compression $[18,21]$.
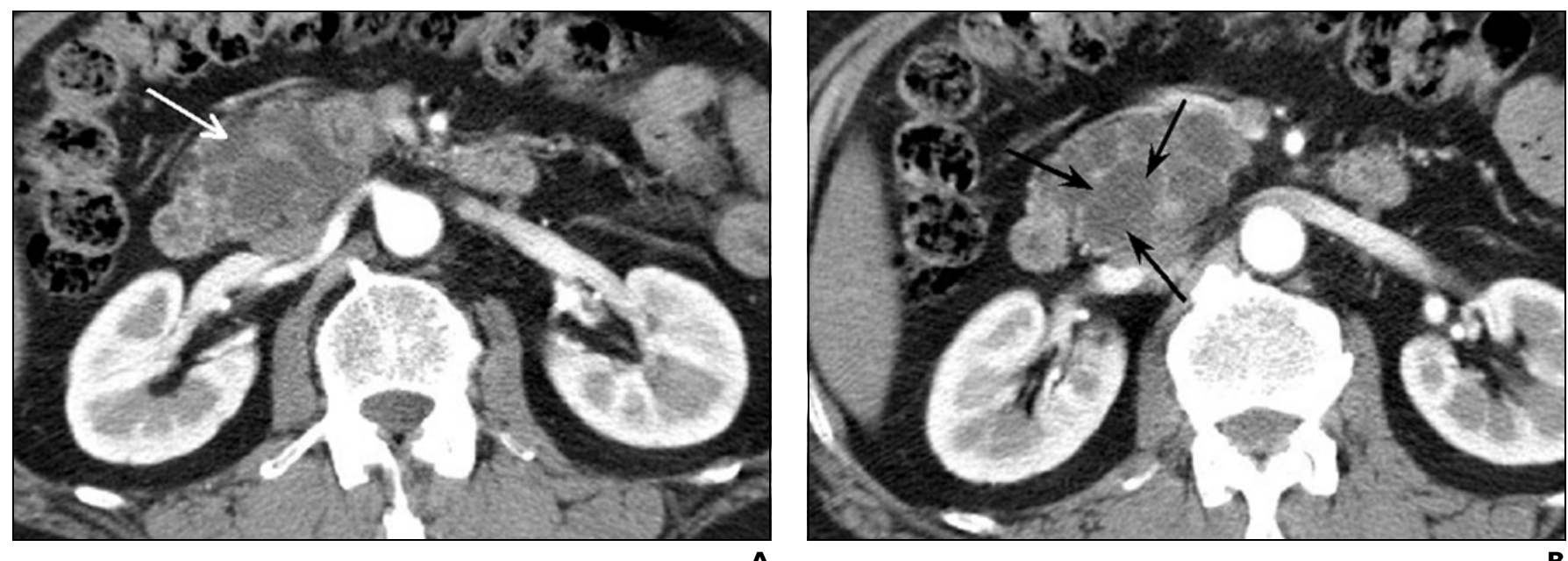

A

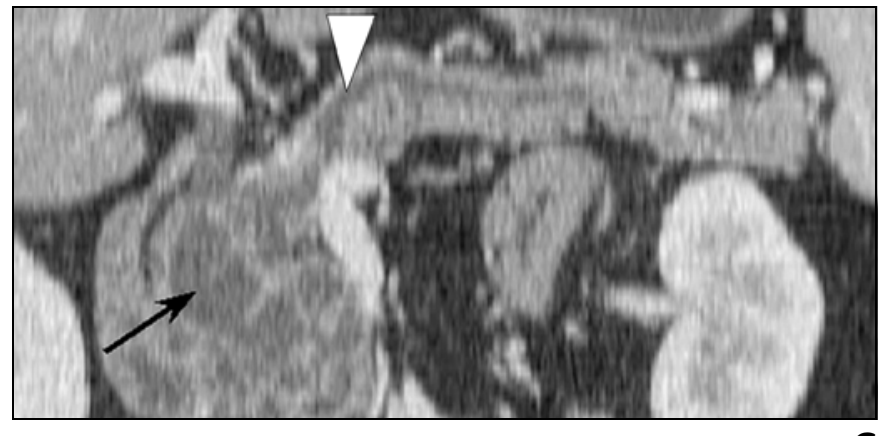

Fig. 5-Branch duct type intraductal papillary mucinous tumor of pancreas in 65year-old man.

A and B, Contrast-enhanced CT scans obtained during pancreatic (A) and portal venous (B) phases show grapelike cystic mass in head of pancreas (arrow, A). Mass contains variable cysts such as oval and tubular cysts (arrows, B) and is of typical pleomorphic cystic shape.

C, Maximum-intensity-projection image reconstructed in coronal plane shows variable cysts (arrow) and whole main pancreatic duct dilatation (arrowhead).
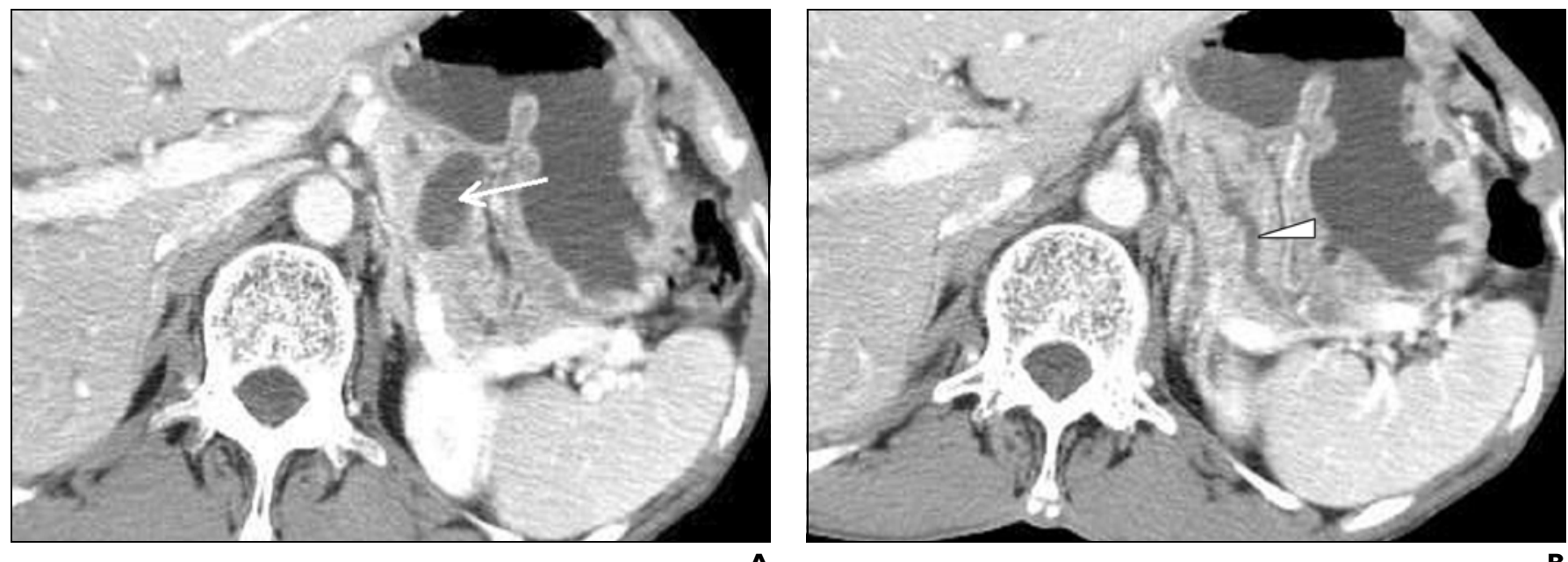

Fig. 6-Branch duct type intraductal papillary mucinous tumor of pancreas in 64-year-old man.

$\mathbf{A}$ and $\mathbf{B}$, Contrast-enhanced CT scans obtained during pancreatic phase show clubbed fingerlike cystic mass in tail of pancreas (arrow, A). Dilatation of entire main pancreatic duct (arrowhead, B) was also noted. 


\section{Kim et al.}

TABLE 3: Significant CT Features for Differentiating Serous Cystadenomas from Other Cystic Neoplasms

\begin{tabular}{|c|c|c|c|}
\hline Type & Shape & $\begin{array}{l}\text { Specificity (\%) } \\
\text { of Shape }\end{array}$ & MPD Dilatation \\
\hline Serous oligocystic adenoma & Multicystic, lobulated & 90 & $\begin{array}{l}\text { None } \\
\text { If dilated, proximal only }\end{array}$ \\
\hline Mucinous cystadenoma & Smooth & 92.2 & $\begin{array}{l}\text { Statistically inadequate } \\
\text { for differentiation }\end{array}$ \\
\hline Intraductal papillary mucinous tumor & $\begin{array}{l}\text { Pleomorphic, clubbed } \\
\text { fingerlike }\end{array}$ & 77.8 & If dilated, whole \\
\hline
\end{tabular}

Note-MPD = main pancreatic duct.

In previous reports [7, 13], wall calcification was considered to be more frequent in serous cystic tumors than in mucinous cystadenomas. However, in our study, no case of serous oligocystic adenoma showed wall calcification. On the other hand, mucinous cystadenoma and intraductal papillary mucinous tumor had some calcified walls. Thus, wall calcification was not found to be a useful feature for the differentiation of the three diseases. In addition, several previous studies $[14,16,19]$ reported that lesion location and patient sex and age may be distinguishing features for these three disease entities at diagnosis. In our study, these features did not show statistical significance. However, because our study population was small, these findings must be confirmed by a large series.

Our study has several limitations. First, this was a retrospective study and was subject to all biases affecting such studies, including that radiologic-pathologic correlation was not possible. Second, different CT scanners were used, and therefore CT parameters are not the same in each patient (5-mm detector collimation in single-detector CT vs $2.5 \mathrm{~mm}$ in MDCT). Last, the number of patients who were enrolled in this study was small. Because MDCT provides improved spatial resolution that allows goodquality multiplanar reformation compared with single-detector $\mathrm{CT}$, further study in large population using MDCT will be warranted.

In conclusion, serous oligocystic adenoma of the pancreas has typical CT findings that differ from other macrocystic neoplasms such as mucinous cystadenoma and intraductal papillary mucinous tumor. The typical imaging features of serous oligocystic adenoma are either a multicystic or a lobulated cystic lesion with or without internal septation (specificity, 90\%). The typical imaging features of mucinous cystadenoma are a smooth cystic lesion with or without internal septation, and those of intraductal papillary mucinous tumor are either a pleomorphic cystic or clubbed, fingerlike cystic lesion. In terms of MPD dilatation, if MPD dilatation is detected, serous oligocystic adenoma has proximal MPD dilatation on CT.

1. Egawa N, Maillet B, Schroder S, Mukai K, Kloppel G. Serous oligocystic and ill-demarcated adenoma of the pancreas. Virchows Arch 1994; 424:13-17

2. Inoue S, Yamaguchi K, Shimizu S, et al. Serous cystadenoma of the pancreas with atypical imaging features: a new variant of serous cystadenoma of the pancreas? Pancreas 1998; 16:102-106

3. Le Borgne J, de Calan L, Partensky C. Cystadenomas and cystadenocarcinomas of the pancreas: a multi-institutional retrospective study of 398 cases. French Surgical Association. Ann Surg 1999; 230:152-161

4. Bassi C, Salvia R, Molinari E. Biasutti C, Falconi M, Pederzoli P. Management of 100 consecutive cases of pancreatic serous cystadenoma: wait for symptoms and see at imaging or vice versa? World J Surg 2003; 27:319-323

5. Procacci C, Carbognin G, Biasiutti C, et al. Serous cystadenoma of the pancreas: imaging findings. $R a$ diol Med (Torino) 2001; 102:23-31

6. Kruslin B, Zovak M, Doko M, Belicza M. Serous oligocystic and ill-demarcated adenoma of the pancreas. Virchows Arch 2002; 440:441-442

7. Warshaw AL, Compton CC, Lewandrowski K, Cardenosa G, Mueller PR. Cystic tumors of the pancreas: new clinical, radiologic, and pathologic observations in 67 patients. Ann Surg 1990; 212:432-443

8. Ooi LL, Ho GH, Chew SP, Low CH, Soo KC. Cystic tumours of the pancreas: a diagnostic dilemma. Aust N Z J Surg 1998; 68:844-846

9. Capella C, Solcia E, Kloppel G, Hruban RH. Serous cystic neoplasms of the pancreas. In: Hamilton SR,

\section{References}

Aaltonen LA, eds. Pathology and genetics of tumor of the digestive system: WHO classification of tumors. Lyon, France: IARC Press, 2000:231-233

10. Lewandrowski K, Warshaw A, Compton C. Macrocystic serous cystadenoma of the pancreas: a morphologic variant differing from microcystic adenoma. Hum Pathol 1992; 23:871-875

11. Compagno J, Oertel JE. Microcystic adenomas of the pancreas (glycogen-rich cystadenomas): a clinicopathologic study of 34 cases. Am J Clin Pathol 1978; 69:289-298

12. Warshaw AL, Compton CC, Lewandrowski K, Cardenosa G, Mueller PR. Cystic tumors of the pancreas: new clinical, radiologic, and pathologic observations in 67 patients. Ann Surg 1990; 212:432-443

13. Procacci C, Graziani R, Bicego E, et al. Serous cystadenoma of the pancreas: report of 30 cases with emphasis on the imaging findings. J Comput Assist Tomogr 1997; 21:373-382

14. Cohen-Scali F, Vilgrain V, Brancatelli G, et al. Discrimination of unilocular macrocystic serous cystadenoma from pancreatic pseudocyst and mucinous cystadenoma with CT: initial observations. Radiology 2003; 228:727-733

15. Curry CA, Eng J, Horton KM, et al. CT of primary cystic pancreatic neoplasms: can CT be used for patient triage and treatment? AJR 2000; 175:99-103

16. Chatelain D, Hammel P, O'Toole D, et al. Macrocystic form of serous pancreatic cystadenoma. Am J Gastroenterol 2002; 97:2566-2571

17. Taouli B, Vilgrain V, O'Toole D, Vullierme MP, Terris B, Menu Y. Intraductal papillary mucinous tumors of the pancreas: features with multimodality imaging. J Comput Assist Tomogr 2002; 26:223-231

18. Hong TM, Lee RC, Chiang JH, Chang CY. Intraductal papillary mucinous tumor of the pancreas: computerized tomography and magnetic resonance imaging features. Kaohsiung J Med Sci 2003; 19:55-61

19. Khurana B, Mortele KJ, Glickman J, Silverman SG, Ros PR. Macrocystic serous adenoma of the pancreas: radiologic-pathologic correlation. AJR 2003; 181:119-123

20. Santos LD, Chow C, Henderson CJ, et al. Serous oligocystic adenoma of the pancreas: a clinicopathologic and immunohistochemical study of three cases with ultrastructural findings. Pathology 2002; 34:148-156

21. Maguchi H, Takahashi K, Katanuma A, Hayashi T, Yoshida A, Sakurai Y. Intraductal papillary mucinous tumor: imaging diagnosis [in Japanese]. Nippon Geka Gakkai Zasshi 2003; 104:447-452 